\title{
BMJ Open University population-based prospective cohort study of SARS- CoV-2 infection and immunity (SARSSURV-ULiège): a study protocol
}

\begin{abstract}
Anne-Françoise Donneau (D) , ${ }^{1}$ Michèle Guillaume, ${ }^{1}$ Vincent Bours, ${ }^{2}$ Margaux Dandoy, ${ }^{3}$ Gilles Darcis, ${ }^{4}$ Daniel Desmecht, ${ }^{5}$ Anh Nguyet Diep (D) , 1 Laurence Fievez, ${ }^{6}$ Mutien-Marie Garigliany, ${ }^{5}$ Nicolas Gillain, ${ }^{1}$ Eddy Husson, ${ }^{1}$ Fabienne Michel, ${ }^{7}$ Michel Moutschen, ${ }^{4}$ Marine Paridans, ${ }^{8}$ Pétre Benoît, ${ }^{8}$ Catherine Sabatel, ${ }^{6}$ Claude Saegerman, ${ }^{9}$ Amandine Tytgat, ${ }^{6}$ Laurent Gillet, ${ }^{10}$ Fabrice Bureau ${ }^{6}$
\end{abstract}

To cite: Donneau A-F, Guillaume M, Bours V, et al. University population-based prospective cohort study of SARS-CoV-2 infection and immunity (SARSSURV-ULiège): a study protocol. BMJ Open 2022;12:e055721. doi:10.1136/ bmjopen-2021-055721

- Prepublication history for this paper is available online. To view these files, please visit the journal online (http://dx.doi org/10.1136/bmjopen-2021055721).

LG and FB contributed equally.

A-FD and $M G$ are joint first authors.

Received 21 July 2021 Accepted 22 December 2021

Check for updates

(C) Author(s) (or their employer(s)) 2022. Re-use permitted under CC BY-NC. No commercial re-use. See rights and permissions. Published by BMJ.

For numbered affiliations see end of article.

Correspondence to Professor Anne-Françoise Donneau; afdonneau@uliege.be

\section{ABSTRACT}

Introduction For a safe and sustainable return to normal functioning of academic activities in higher education, objective-driven testing strategies that are flexible and rapidly adaptable are essential to effectively monitor and respond to new developments of the COVID-19 pandemic. To date, prospective longitudinal research on SARS-CoV-2 antibody testing in saliva and seroprevalence in higher education contexts is substantially lacking, limiting our understanding of COVID-19 prevalence, incidence and nature of the immune response to SARS-CoV-2 at various stages of the infection and vaccination. To address this lack of evidence, a prospective population-based cohort study (SARSSURV-ULiège) has recently been started. Methods and analysis Students $(n=1396)$ and staff members $(n=1143$ ) of the University of Liège are followed up over more than 1 year. All participants are required to complete anamnestic, clinical and vaccine hesitancy questionnaires for medical histories and undertaken treatments. Previous proven or suspected SARS-CoV-2 infection is also registered. In phase 1, weekly saliva samples to perform RT-qPCR to detect SARS-CoV-2 and monthly COVID-19 serological rapid test results are collected. Once being positive to either saliva RT-qPCR assay for SARS-CoV-2 presence or to serological test, the participant is invited to enter phase 2. If participants get vaccinated during the study period, they are invited to phase 2 . In this second phase, besides weekly saliva self-test, depending on the participants' profiles, both gargle and blood samples are collected to obtain various biological data to measure the presence of neutralising antibodies against SARS-CoV-2, determine the magnitude and the duration of antibody responses over time.

Ethics and dissemination The study has received the approval from the University Hospital of Liège Ethics Committee (reference number 2021/96, dated 26 March 2021). Potential protocol amendments will be presented to the Research Ethics Committee. The findings of the present study will be presented at scientific conferences and the results published in peer-review publications. Weekly reports will be submitted to the risk assessment

\section{Strengths and limitations of this study}

- This is the first European university populationbased cohort study that aims to study SARS-CoV-2 infection, immune response to SARS-CoV-2 infection and vaccine, and vaccination hesitancies.

- The prospective design of SARS-CoV-2 Survey (SARSSURV) allows to derive reliable estimates of the prevalence, the incidence of COVID-19 in both symptomatic and asymptomatic participants, characterising the immune response while accounting for vaccine and/or previous infection a in higher education population.

- The longitudinal study design enables to derive these results at different stages of the disease as well as seroreversion and seroconversion after vaccination, hence informed decisions as to COVID-19 measures and resuming of activities being further strengthened.

- The inclusion of participants may be biased by their engagement against COVID-19, which may result in low secondary infection as participants could take more precaution against COVID-19 transmission.

- Given the longitudinal design of the study and a recall system in place, the presence of intermittent missing data and drop-out are unavoidable.

group and the risk management group against COVID-19 of the university to enable a timely public health action if necessary.

\section{INTRODUCTION}

Infection with SARS-CoV-2 induces COVID19. Since the first report of the SARS-CoV-2 in December 2019, in Wuhan, China, COVID-19 has been recognised on 11 March 2020, by the $\mathrm{WHO}$ as a pandemic. ${ }^{1}$ At the moment, a total of 184252078 confirmed COVID-19 cases and 3986701 deaths related to 
COVID-19 have been reported worldwide. ${ }^{2}$ In Belgium, the first COVID-19 confirmed case was registered on 2 March 2020. ${ }^{3}$ Mid-March 2020, in the absence of treatment and vaccine options, the Belgium authorities implemented the first restrictions to individual movement. To control the transmission of COVID-19, those restrictions have evolved regularly according to some indexes, such as COVID-19 testing positivity rate and the occurrence of virus variants of concern. As of 20 June 2021, in Belgium, 1088363 confirmed COVID-19 cases and 25190 deaths have been registered, making it among the 10 countries in the world with most deaths per 1 million population. ${ }^{4}$

SARS-CoV-2 seems transmitted among people via direct human contact and infectious respiratory droplets. ${ }^{5-7}$ Therefore, based on these modes of transmission, SARS-CoV-2 can spread sharply as a consequence of noncompliance to strict hygiene protocols, societal protective measures like face mask wearing and physical distancing, and proper room ventilation. In these perspectives, reopening universities in September 2020 was a real challenge because the teaching and learning activities entailed more travel time and face-to-face contact of longer hours, which meant these rules of social distancing and protective measures were at stake. In other words, university campuses are viewed as dense social networks where the likelihood of community transmission and outbreaks is not negligible ${ }^{8}$ and should be mitigated to ensure normal functioning. In this regard, objective-driven testing strategies that are flexible, rapidly adaptable and sustainable are advocated by the European Center for Disease Prevention and Control in order to effectively monitor and respond to new developments of the pandemic. ${ }^{9}$ Thus, massive and systematic screening of SARS-CoV-2 using saliva and blood samples and/or any other easily accessible samples and tracking seroprevalence for infection over time is essential to promptly detect cases of infection, which serves as a basis for informed decision making regarding teaching and learning organisation in higher education. More specifically, performing massive and systematic screening of SARS-CoV-2 using saliva and blood samples and tracking seroprevalence for infection over time in two higher education populations (students and staff members) of diverse backgrounds and worklife circumstances can help capture the dynamics of viral transmission, seroreversion and seroconversion in both symptomatic and asymptomatic individuals. This has several implications for research and practices.

As observed, not all SARS-CoV-2 positive cases are symptomatic, particularly in healthy and young populations. ${ }^{10}$ Furthermore, due to limited testing capacity, only a proportion of COVID-19 suspected individuals have access to invasive tests such as blood-based diagnostic specimens. Non-invasive SARS-CoV-2 detecting and antibody testing in saliva samples have been explored as an alternative to arrange massive testing so as to rapidly detect COVID-19 positive cases. ${ }^{11}{ }^{12}$ Current evidence has supported the efficacy of saliva testing in the detection of SARS-CoV-2 compared with other tests including the dominating nasopharyngeal swab sampling. ${ }^{13}{ }^{14}$ In a systematic review and meta-analysis on 16 unique studies extracted up to November 2020, the pooled sensitivity and specificity of saliva testing were $83.2 \%$ (95\% credible interval: $74.7 \%-91.4 \%)$ and $99.2 \%$ (95\% credible interval: $98.2 \%-99.8 \%$ ), respectively, in comparison with a pooled sensitivity of $84.8 \%$ (95\% credible interval: $76.8 \%-92.4 \%)$ and specificity of $98.9 \%$ (95\% credible interval: $97.4 \%-99.8 \%) .{ }^{15}$ The findings, hence, suggested a similar efficacy of saliva testing as opposed to nasopharyngeal swab testing and supported large-scale research employing saliva testing. In addition to the documented similar sensitivity, repeated sampling revealed that despite a decreased level of SARS-CoV-2 RNA in both saliva and nasopharyngeal swab specimens after symptom onset, less variation in the levels of SARS-CoV-2 RNA was observed in the former. ${ }^{16}$ In asymptomatic patients, the false negative rate was found to be higher in nasopharyngeal swab than in saliva specimens, which could be attributed to the variation in nasopharyngeal sampling. ${ }^{16}$ However, for saliva testing to be yield acceptable and comparable sensitivity with that of nasopharyngeal swabs, a standardised method of saliva sample collection and RNA extraction is strongly recommended. ${ }^{13}$

Another body of research focuses on seromonitoring ${ }^{12}$ in order to examine the heterogeneity and seroreversion among SARS-CoV-2 infected cases of different levels of disease severity, all of which contributes to the global joint effort to effectively manage the pandemic. Most studies of seroprevalence were conducted retrospectively among the general population, blood donors, factory workers or local community residents or based on residual specimens from laboratory blood tests. Prospective longitudinal research on SARS-CoV-2 antibody testing in saliva and seroprevalence in a higher education context is substantially lacking. This has limited our understanding of seroprevalence and immune response to the SARSCoV-2 and the different COVID-19 vaccines over time, particularly in a higher education population characterised by frequent and close contacts during the teaching and learning as well as social activities. In light of vaccination programmes that have been activated recently across different countries and territories, the significance of longitudinal research on COVID-19 prevalence, incidence and nature of the immune response to the SARSCoV-2 is essentially justified.

\section{STUDY AIMS AND SIGNIFICANCE}

Designed as a population-based cohort study including students and staff members of the University of Liège (ULiège), our aims are to investigate the seroprevalence, the immune response to SARS-CoV-2 at various stages of the infection and after vaccination. More specifically, the present study will collect saliva and blood samples to achieve the following research objectives (ROs) and address the following research questions (RQs). 
RO1. To capture the epidemiological evolution in the ULiège population during the time of the study:

- RQ1: what is the prevalence of SARS-CoV-2 within the university on a monthly basis?

- RQ2: what is the incidence of SARS-CoV-2 within the university on a monthly basis?

RO2. To investigate the nature of the immune response to the SARS-CoV-2 vaccine (ie, the titre of the anti-SARSCoV-2 antibody, their capacity to neutralise the virus (seroneutralisation titers) and the persistence this antibody response over time):

- RQ3: what is the titre of the anti-SARS-CoV-2 antibody and the titre of the SARS-CoV-2 neutralising antibody after SARS-CoV-2 vaccine?

- RQ4: is the nature of the immune response to SARSCoV-2 vaccine comparable with the response triggered after SARS-CoV-2 infection?

- RQ5: what is the efficacy of the serological rapid self-test?

RO3. To longitudinally monitor the persistence and nature of the immune response and factors associated with the intention and decision to get vaccinated against COVID-19, in different target groups:

- RQ6: is the immune response after vaccination persistent?

- RQ7: is the response to the vaccine different in previously infected individuals in terms of antibody development and levels over time?

- RQ8: what are the factors associated with the participants' attitudes, beliefs, intentions and decisions to get vaccinated against COVID-19?

- RQ9: to what extent do the participants' attitudes, beliefs, intentions and decisions to get vaccinated against COVID-19 change over time?

RO4. To investigate the effect of SARS-CoV-2 vaccine:

- RQ10: can the vaccine prevent carriage of SARS-CoV-2?

RO5. To longitudinally monitor the COVID-19 disease progression employing duration and symptoms as main clinical indicators:

- RQ11: what is the duration of SARS-CoV-2 positivity?

- RQ12: what is the evolution of symptoms and/or side effects in case of SARS-CoV-2 infection?

- RQ13: what are the symptoms and/or side effects related to the different vaccines?

Performing massive and systematic screening of SARSCoV-2 using saliva and blood samples and tracking seroprevalence for infection over time in two higher education populations (students and staff members) of diverse backgrounds and work-life circumstances can help capture the dynamics of viral transmission, seroreversion and seroconversion in both symptomatic and asymptomatic individuals. This has several implications for research and practices. First of all, the evidence will enhance our knowledge of the nature of viral transmission, immunity, seroreversion, seroconversion and effectiveness of COVID-19 vaccination programme. Second, particularly in the Belgian context, the results obtained will enable prompt decision makings to mitigate the transmission scale, evaluate currently imposed measures and resume normal teaching and learning activities that have been greatly affected and suspended since the announcement of a global COVID-19 pandemic.

Against this background, this paper documents a protocol for a university population-based prospective cohort study of SARS-CoV-2 infection and immunity (SARSSURV-ULiège).

\section{METHODS AND ANALYSIS \\ Design}

SARSSURV-ULiège is a prospective observational cohort study of students and staff members from the University of Liège, Belgium. Both, students and staff members, are followed up from April 2021 to June 2022. Indeed, the purpose of the present study is to collect weekly saliva samples to perform quantitative real-time reverse transcriptase PCR (RT-qPCR) to detect SARS-CoV-2 and monthly COVID-19 serological rapid test results (phase 1 ) over a period of at least 8 months. When being positive to either saliva RT-qPCR assay for SARS-CoV-2 presence or to serological test, the participant is invited to enter phase 2 of the study. In this second phase, depending on the participant's profile (see 'Participant pathway'), both gargle sample and blood samples are collected. If a participant gets vaccinated during the study period, he or she is invited to join phase 2 .

\section{Study population}

In order to constitute SARSSURV-ULiège cohorts, a representative sampling procedure according to the age, gender and assignment/faculty proportional to the ULiège population is applied to both students and staff members.

\section{Recruitment procedure}

All university members meeting the study criteria receive a personalised invitation sent by means of the university internal mailing system. As the objective is to constitute a cohort that could be followed until the next academic year, the study criteria are as follows.

\section{Inclusion criteria}

1. Be part of one of the following two cohorts of individuals: staff members or students of the ULiège.

2. Have provided an online informed consent.

\section{Exclusion criteria}

For staff members: have a working contract which ended before 31 December 2021.

For students: be registered in first or last year of cursus, or in other courses and/or programmes that can potentially be completed in the current academic year or be $\mathrm{PhD}$ students who could potentially defend the $\mathrm{PhD}$ thesis before the end of 2021.

Prior to the personalised invitation mail, the university population was informed about the present study through a mail from the rector, as well as through various 


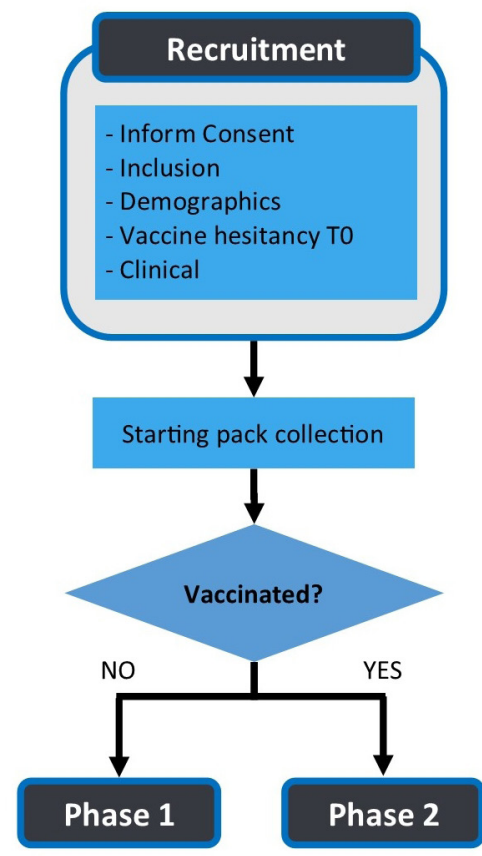

Figure 1 Participant pathway - recruitment procedure SARSSURV-ULiège cohort study.

internal communications including videos and ULiège internal news. ${ }^{17}$

Regarding age restrictions, participants younger than 18 years or older than 67 years, that is, the age of retirement specified by law in Belgium, were not eligible to participate. In so doing, the sample would maximally mimic the ULiège population.

Volunteers interested to participate in the study are invited to register via an online platform using the personalised link available in the invitation mail. The pathway of the participants from enrolment until end of the study is detailed in next section.

If a participant does not meet the inclusion criteria, they are encouraged to continue their investment against COVID-19 by remaining vigilant, applying barrier gestures and performing the weekly saliva testing offered by the university.

\section{Participant pathway}

The detailed pathway of the participants from recruitment until end of the study is depicted in figures 1 and 2 , respectively, with the details explained in next sections that follow.

\section{Consent procedure}

Online informed consent is collected from each participant. Personal identifying information, including the consent date, sample results and questionnaire responses are stored in a crypted database. Compliance with data protection regulations is approved by the official data protection officer of the University of Liège. The data are stored for as long as they are required to achieve the study objectives.

\section{Procedure}

After online consent forms have been signed, participants are invited to provide identification information and to select the place where they can collect their starting pack, composed of saliva self-sampling devices, serological rapid self-tests, gargle kit (physiological liquid +collecting pot), identification tags, as well as written practical instructions for use of all delivered self-tests. Finally, to complete their registrations, they have to fill in two questionnaires, one anamnestic and clinical questionnaire, and a COVID-19 vaccine hesitancy, both described here further. At the end of the inclusion step, a unique identification code (ID) is attributed to each participant.

\section{Anamnestic and clinical questionnaires}

A short questionnaire is proposed to collect medical history and possible treatments undertaken by participants. In particular, previous proven or suspected SARS$\mathrm{CoV}-2$ infection is registered.

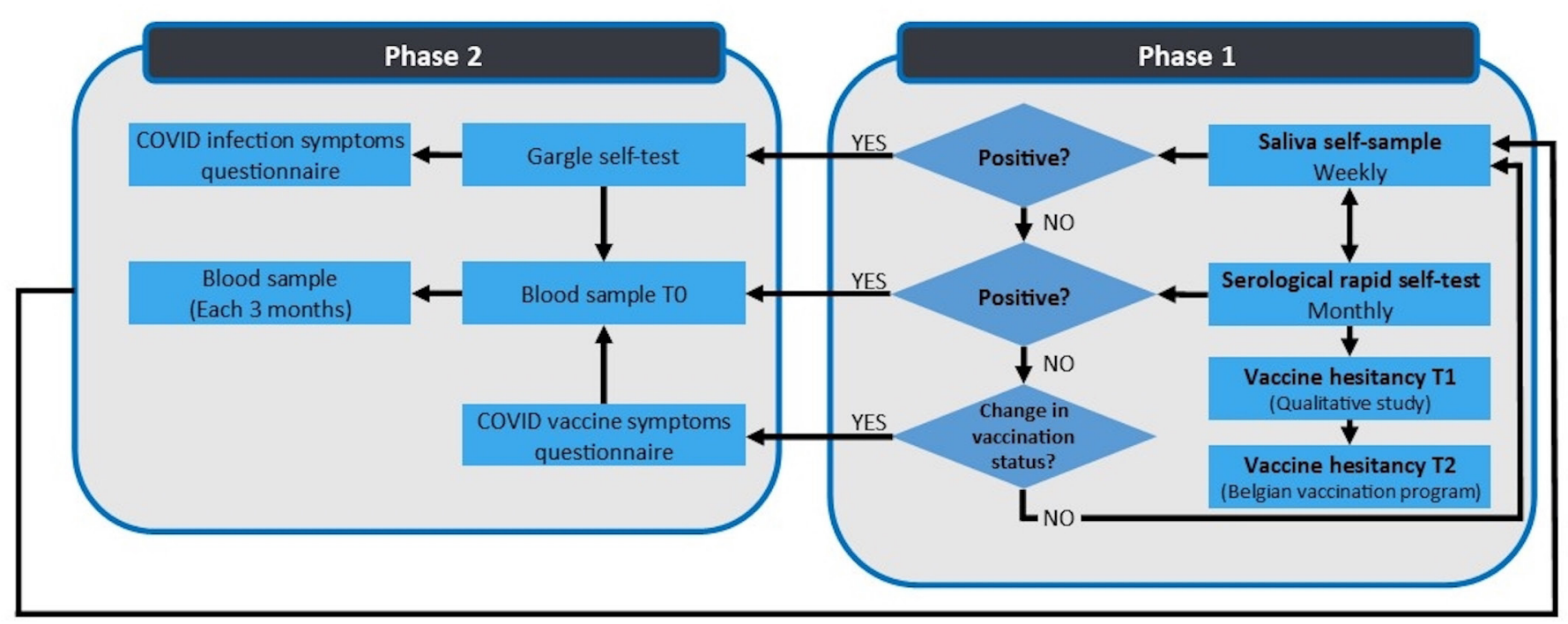

Figure 2 Participant pathway - follow-up procedure SARSSURV-ULiège cohort study. 
Vaccine hesitancy questionnaire

Vaccine hesitancy is not a new phenomenon and has been identified by the WHO as one of the 10 threats to global population health. From a public health standpoint, understanding vaccine hesitancy is a key issue in supporting approaches to promote population autonomous and informed choice. Previous research has documented factors related to vaccine hesitancy. ${ }^{18-20}$ The classification of the Strategic Advisory Group of Experts on Immunization suggests three categories of factors ${ }^{20}$ : context-related determinants (communication, influence of leadership and groups, geographic accessibility, etc), individual-related and group-related determinants (past vaccination experience, attitudes towards health (prevention), attitudes towards the vaccine, knowledge awareness, perception of risks and benefits, trust in health professionals, etc) and vaccine-specific determinants (risks and benefits, new vaccine, vaccine scheduling, etc). Multivariate analysis of the data should allow identification and weighting of the determinants of vaccine hesitancy in the specific context of COVID-19.

The COVID-19 vaccine acceptance and hesitancy questionnaire is divided into several parts including questions on respondents' characteristics, past vaccination experience, COVID-19 experience, health and engagement in health, personality, beliefs, attitudes, perceptions and knowledge about COVID-19 vaccination, vaccination decision and intention and future vaccination. During the study period, participants will be contacted to participate in the second COVID-19 vaccine survey phase, which will be a qualitative approach in order to better understand the mechanisms underlying vaccine intention taking into account the evolution of the sanitary and vaccine situation in Belgium. In this regard, the perspectives concerning vaccination intention at different stages of the data collection will be coupled with governmental reports on the COVID-19 pandemic evolution, vaccination rate, and vaccination intention, particularly in the Walloon Region, where the research was conducted. A last questionnaire will be organised in the fall after the Belgian vaccination programme is completed.

\section{Phase 1}

This first phase of the study is achieved by all participants from enrolment up to end of June 2022. The different steps of phase 1 are presented hereafter.

\section{Saliva self-sample}

Participants have to perform a weekly saliva self-sample to detect the presence of SARS-CoV-2 following the instruction provided in the starting pack. The collected saliva sample is dropped off by the participant, the same day, in one of the dedicated collection stands. Participants are instructed to consult, within 12 hours of sample receipt at the stand, saliva self-test result on the ULiège platform. ${ }^{21}$ The test result could be negative, positive or uninterpretable. In a limited number of cases, the analysis cannot be performed (non-compliant sample).
From a practical point of view, in addition to the written detailed procedure provided with the starting kit, a video is also available. ${ }^{22}$

\section{Serological rapid self-test}

Participants are also instructed to perform, once a month, a serological rapid self-test to detect the presence of immune response to the SARS-CoV-2. This is a self-test that can be done very easily (at home) by taking a drop of blood from the tip of the finger, in a way that is quite similar to measuring the blood glucose level of a diabetic patient. Instruction about how to perform the serological rapid self-test are provided in the starting pack. The test result could be negative, positive or invalid. The response on the patient's immune status is directly visible by the participant on the kit within $10 \mathrm{~min}$. Participants have then to report the corresponding results during their consultation of salivary test result.

\section{Phase 2}

If a positive result occurs during phase 1 for saliva or serological rapid self-test, concerned participants are contacted by members of the research team to be informed that they enter in phase 2 of the study. In the same way, participants who get vaccinated during the course of the study also enter phase 2. Indeed, vaccination should induce the production of antibodies that will be detected by the serological rapid self-test. As a reminder, participants are requested to report possible changes in their vaccination status during weekly saliva result consultation.

While a quarantine period will be respected prior to phase 2 steps for participants with a positive SARSCoV-2 infection, participants with a positive anti-SARSCoV-2 immune response will directly enter phase 2 . The different steps of phase 2 are presented hereafter.

\section{Saliva self-sample}

In phase 2, participants have to pursue saliva self-test once a week, using the same scheme as in phase 1 . This weekly saliva self-test in phase 2 is very important to detect new infection, regardless of the participant's vaccination or serological status. These new infections could multiply with the arrival of some new variants that could escape the host immune system and the vaccine response. ${ }^{23}$

\section{Gargle self-test}

In case a positive result is obtained for the saliva selftest, it is subject to verification by performing a gargle sampling on the next morning before eating and subsequent RT-qPCR, sequencing and isolation tests. This gargle self-sample realised by the participant is collected at the participant's home to ensure that the quarantine period is well respected. The purpose of the gargle selftest is to attempt viral isolation. Results of gargle sample analysis are encoded in the online study platform by the laboratory in charge of the analysis. 


\section{Blood samples}

In order to measure the ability of antibodies to neutralise SARS-CoV-2, blood samples are planned every 3 months by nurses of the research team under the responsibility of the investigating physician at the participant's home or at specific rooms in the university campus (currently, five open spots). For participants with a positive COVID-19 infection (ie, positive saliva self-test), blood samples are planned after the quarantine period and 3 months after. The blood samples are planned at entry in phase 2 and 3 months thereafter, for participants who either have a positive result for the serological self-test or get COVID-19 vaccinated. The samples are stored at $4^{\circ} \mathrm{C}$ and transported to the laboratory. The purpose of the blood sample is to collect various biological data to measure the presence of neutralising antibodies against SARSCoV-2 and to determine the magnitude and the duration of antibodies responses over time. Results of the blood samples are encoded in the online platform by the laboratory members in charge of the analysis. Finally, the result (level of immunity) is communicated to the participant by the nurses of the research team under the responsibility of the investigating physician.

\section{COVID-19 vaccine symptoms and/or side effects}

Nurses of the research team collect participant's symptoms and/or side effects related to vaccination during the course of the study. Those symptoms are collected during face-to-face interview at the same time of the first blood sample related to the change in the vaccine status of the participant. A total of 13 symptoms suggestive of COVID-19 vaccination are considered: fatigue, headaches, loss of appetite, myalgias, confusional syndrome, nausea, vomiting, fever, arthralgias (joint pain), pain at the injection site, axillary adenopathy (swollen lymph node on the side of the injection site), redness at the injection site, allergic reaction and others. For each symptom and/or side effect, the result (Likert scale ranging from 0 (no symptom) to 10 (severe symptom)) is encoded in the online platform by the nurses.

\section{COVID-19 infection symptoms and swab (on a voluntary basis)}

In case of a positive result for the saliva self-test, the following additional steps are proposed to the participants on a voluntary basis. Nurses of the research team visit the participant twice a week during a period of 3 weeks in order to realise a nasopharyngeal swab for COVID-19, as well as a saliva self-test. In addition, during this visit, the nurse collects participant's symptoms related to COVID-19 infection. In case the participant does not agree for swab voluntary study, COVID-19 infection symptoms are still collected by phone. A total of 20 symptoms suggestive of SARS-CoV-2 infection are considered: dyspnoea effort, fatigue, dry cough, chest pain, headache, loss of appetite, myalgias, dyspnoea at rest, anosmia, agueusia, rhinorrhea, paresthesias/dysesthesias, memory loss, diarrhoea, wet cough, pharyngeal pain, confusional syndrome, nausea, vomiting, fever and other. ${ }^{24-26}$ For each symptom,

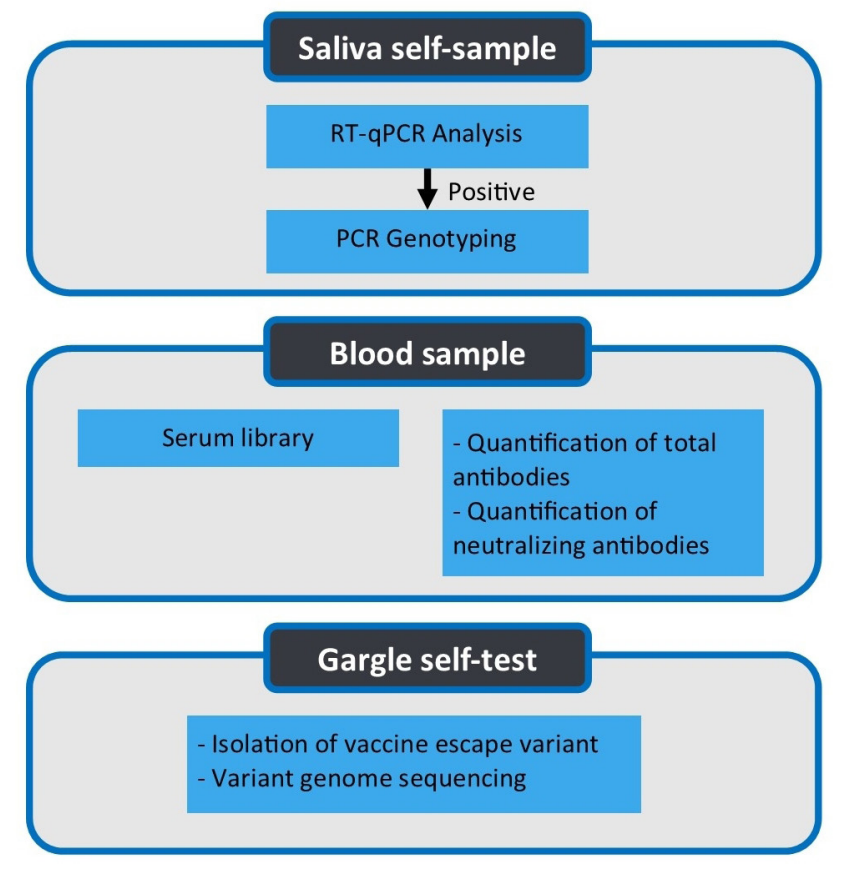

Figure 3 Pathway of human samples, SARSSURV-ULiège cohort study.

the result (Likert scale ranging from 0 (no symptom) to 10 (severe symptom) ) are encoded in the online platform by the nurses.

\section{Laboratory analysis}

The detailed pathway of the human samples collected for the study is depicted in figure 3 .

\section{Saliva sampling}

Fresh fasting saliva sample is collected using a dedicated device designed by the University of Liège, commercialised by Diagenode (Seraing, Belgium) and analysed using RT-qPCR method detailed hereafter to detect the presence of the SARS-CoV-2.

\section{RNA extraction from saliva and pooling}

In the collection device, saliva is diluted at a $1: 1$ ratio with an extraction buffer containing $1 \mathrm{M}$ guanidine thiocyanate (GITC). Samples are incubated at $80^{\circ} \mathrm{C}$ for 20 min. All saliva samples are spiked with 16 copies of a purified MS2 bacteriophage (1:40 MS2:sample) prior to RNA extraction. For pooled extractions, RNA extraction is performed on $60 \mu \mathrm{L}$ saliva per sample (pools of 3) plus $170 \mu \mathrm{L}$ lysis buffer containing GITC 4M. Extraction is performed using CoRNA Isolation Kit (Diagenode, Seraing, Belgium) following the manufacturer's protocol and using $50 \mu \mathrm{L}$ of magnetic beads. Extracted RNA is eluted from magnetic beads in $50 \mu \mathrm{L}$ UltraPure DNase/ RNasefree distilled water. For individual extractions, RNA extraction is performed on $100 \mu \mathrm{L}$ saliva per sample plus $300 \mu \mathrm{L}$ lysis buffer containing GITC 4M. Extraction is performed using CoRNA Isolation Kit (Diagenode, Seraing, Belgium) following the manufacturer's protocol and using $50 \mu \mathrm{L}$ of magnetic beads. Extracted RNA is 
eluted from magnetic beads in $50 \mu$ l UltraPure DNase/ RNasefree distilled water.

\section{RT-qPCR assay}

We perform a multiplex RT-qPCR assay using the TaqPath RT-PCR COVID-19 kit (Thermo Fisher A47817) together with the TaqPath 1-step master mix - No ROX (Thermo Fisher CN A28523). This RT-qPCR assay targets three viral genes, ORFlab, $\mathrm{N}$ and $\mathrm{S}$ genes. All the reactions are performed in a $384 \mathrm{w}$ format (final volume of $20 \mu \mathrm{L}$ ) on a QS5 thermocycler (Applied Biosciences, Waltham, Massachusetts, USA). RT-qPCR reactions are prepared as follows: $5 \mu \mathrm{L}$ of $4 \mathrm{X}$ TaqPath Multiplex MasterMix, 1 $\mu \mathrm{L}$ of COVID-19 real-time PCR assay, $6 \mu \mathrm{L}$ of water and $8 \mu \mathrm{L}$ of RNA (samples or controls). TaqPath COVID-19 positive Control (Thermo Fisher A48003, Thermo Fisher, Waltham, Massachusetts, USA) at 25 genomic copies $/ \mu \mathrm{L}$ is used. The RT-qPCR is run using the standard mode, consisting of a hold stage at $25^{\circ} \mathrm{C}$ for $2 \mathrm{~min}, 53^{\circ} \mathrm{C}$ for 10 min, and $95^{\circ} \mathrm{C}$ for $2 \mathrm{~min}$, followed by 40 cycles of a PCR stage at $95^{\circ} \mathrm{C}$ for $3 \mathrm{~s}$ then $60^{\circ} \mathrm{C}$ for $30 \mathrm{~s}$, with a $1.6^{\circ} \mathrm{C} / \mathrm{s}$ ramp up and ramp down rate. Results are analysed with the FastFinder software (Ugentec, Hasselt, Belgium) and are expressed as quantification cycle $(\mathrm{Cq}$ value, ie, the number of cycles required for the quantification of the fluorescent signal to be considered as positive) with the limit of positivity being fixed under a $\mathrm{Cq}$ value of 37. Samples are considered negative when no viral gene could be detected below a $\mathrm{Cq}$ value of 37 and when the MS2 internal control is under a Cq value of 30. All individual samples are pooled by three. If a pool is tested negative, the status of all associated individual samples is considered as negative. If a pool is tested positive or inconclusive, each associated individual sample is retested to identify which one(s) is (are) positive.

\section{Serological rapid self-test}

This monthly self-test is performed by the participant using BIOSYNEX COVID-19 BSS (IgG/IgM) (Biosynex Swiss SA) ${ }^{27}$ This capillary whole-blood IgG-IgM COVID-19 self-test is a serological screening tool, targeting the spike protein Receptor Binding Domain, adapted to be used by the general population. The provided result is qualitative, visually interpretable and available within $10 \mathrm{~min}$. This self-test has a sensitivity of $97.4 \%$ and specificity of $100 \%$, demonstrating high analytical performances, which then allow management of suspected ongoing and past COVID-19 infections. ${ }^{27}$ In addition, this self- test is recommended by the French Ministry of $\mathrm{Health}^{28}$ for both SARS-CoV-2-specific IgG and IgM detection.

\section{Gargle self-test: SARS-CoV-2 virus isolation}

When a saliva sample is positive, a gargle sample is asked the next day and viral isolation is attempted. For the isolation, $50 \mu \mathrm{L}$ serum-free Dulbecco's Modified Eagle Medium (DMEM) is pipetted into columns 2-12 of a 96-well tissue culture plate. One-hundred microlitres of gargle specimens are pipetted into column 1, and then serially diluted twofold across the plate. Vero cells are trypsinised and resuspended in DMEM $+10 \%$ FBS $+2 \times$ penicillin-streptomycin $+2 \times$ antibiotic-antimycotic $+2 \times$ amphotericin B at $2.5 \times 10^{5}$ cells $/ \mathrm{mL}$. One hundred microlitres of cell suspension are added directly to the clinical specimen dilutions and mixed gently by pipetting. The inoculated cultures are grown in a humidified $37^{\circ} \mathrm{C}$ incubator with $5 \% \mathrm{CO}_{2}$ and observed for cytopathic effect (CPE) daily. When CPE is observed, the cell monolayers are scrapped with the back of a pipette tip. Fifty microlitres of the viral lysate are used for total nucleic acid extraction for confirmatory testing and sequencing. Fifty microlitres of virus lysate is used to inoculate a well of a 90\% confluent 24-well plate for virus variant expansion. When no CPE is identified, the procedure is repeated using the culture supernatants from the previous run. Isolation failure is pronounced only after three successive passages without CPE.

\section{Blood samples: assessment of serum neutralising antibodies}

At both time point, $1 \times 10 \mathrm{~mL}$ EDTA tube and $1 \times 10 \mathrm{~mL}$ serum tube are collected. A serum sample from all participants is stored for quantitating neutralising antibody titres. Virus neutralisation test (VNT) is carried out with SARS-CoV-2 strain BetaCov/Belgium/SartTilman/2020/1 in 96-well plates containing confluent Vero E6 cells (ATCC CRL-1586). Seven dilutions are used of each heat-inactivated serum (1:10 to $1: 640-$ corresponding to final testing dilutions 1:20 to 1:1280), allowing testing two samples or controls per plate. In each VNT, a strong, guaranteed positive control serum from the Belgian National Reference Centre (Sciensano) is used. Sera are mixed vol/vol with 100 TCID50/reaction of SARS-CoV-2 virus and incubated at $37^{\circ} \mathrm{C}$ for 1 hour. Then, the serum plus virus mixture is transferred onto the confluent cell monolayer in triplicate. The VNT relies on CPE observation under light microscopy at day 5 pi. Dilutions of serum associated with CPE is considered as negative, while the absence of CPE indicated a complete neutralisation of SARS-CoV-2 inoculum (positive). Virus neutralisation titre is reported as the highest dilution of serum that neutralised CPE in $50 \%$ of the wells.

The remaining plasma is stored for further analysis or ancillarius studies.

\section{Statistical analysis}

Sample size calculation

A power calculation based on a $95 \%$ CI for a proportion was applied to determine the number of subjects to be included in this study. Accordingly, four parameters had be to specified, including the precision, the prevalence, the population size and the coverage probability of the CI, which was chosen as $95 \%$ in the study. The sample size was calculated with the following formula ${ }^{29}$ :

$\mathrm{n}=\mathrm{N} * \mathrm{X} /(\mathrm{X}+\mathrm{N}-1)$, where

$\mathrm{X}=\mathrm{Z}_{\alpha / 2}{ }^{2} * \mathrm{p} *(1-\mathrm{p}) / \mathrm{d}^{2}$, and $\mathrm{Z}_{\alpha / 2}$ is the critical value of the normal distribution at $\alpha / 2$ (eg, for a confidence level 
of $95 \%, \alpha$ is 0.05 and the critical value is 1.96$), d$ is the margin of error and $\mathrm{p}$ is the expected proportion.

As the seroprevalence of SARS-CoV-2 antibodies was unknown among the target population, the conventional value of $50 \%$ was considered. The population of ULiège students meeting the study criteria was estimated to be 11552 students. A total of 977 students should be included in order to estimate the proportion of interest with a precision of $3 \%$. Considering $30 \%$ lost to follow-up, a total of 1396 students should be recruited. The same scheme applied to the ULiège staff population meeting the study criteria $(n=3184)$ indicated that a total of 800 staff members should be considered. In order to also consider possible dropouts (30\%), a total of 1143 participants should be recruited.

The main results of the present study will be reported according to the Strengthening the Reporting of Observational studies in Epidemiology. ${ }^{30}$ The primary endpoints include:

1. Prevalence and incidence of SARS-CoV-2 infection on a monthly basis.

2. Presence of immune response after SARS-CoV-2 and/ or SARS-CoV-2 vaccine over different time points.

The secondary endpoints are:

1. Characterisation of the immune response after SARSCoV-2 and/or SARS-CoV-2 vaccine.

2. Genomic characterisation of SARS-CoV-2.

3. Description of symptoms among positive COVID-19 participants.

4. Prevalence of SARS-CoV-2 infection on a monthly basis and dynamics of COVID-19 vaccine hesitancy.

Each endpoint will be summarised numerically, with corresponding 95\% CI. Association with environmental, demographics, clinical and behavioural risk factors will be investigated, from a univariate end multivariate point of view, using appropriated statistical analysis methods. Baseline data as well as longitudinal data will be considered for analysis. Statistical analysis will be done using SAS (V.9.4) and R statistical software.

\section{Patient and public involvement}

The participants, namely students and staff members of ULiège, were not involved in the design, recruitment or choice of outcome measures of this research protocol.

\section{ETHICS AND DISSEMINATION}

The study has received the approval from the University Hospital of Liège Ethics Committee (reference number 2021/96, dated 26 March 2021). Potential protocol amendments will be presented to the Research Ethics Committee. The findings of the present study will be presented at scientific conferences and the results published in peer-review publications. Weekly reports will be submitted to the risk assessment group and the risk management group against COVID-19 of the university to enable a timely public health action if necessary.

\section{DISCUSSION}

To the best of our knowledge, SARSSURV is the first European university population-based cohort study that aims to study SARS-CoV-2 infection, immune response to SARS-CoV-2 infection and vaccine, and vaccination hesitancies. Monitoring COVID-19 prevalence, incidence and immune response to SARS-CoV-2 among students and staff members provides an important and unique epidemiological basis to better understand the dynamics of COVID-19. The study is of prime significance in the context when vaccination campaign is widely implemented to enhance herd immunity, hence advancing our resuming of academic activities.

In addition to effort to realise a representative sample and ensure immediate laboratory testing results promptly communicated to the participants during the course of the study, the present study relies significantly on periodic self-sampling, particularly saliva sampling in both phases of the research. In this respect, research has shown that saliva sampling and pooling is a viable and non-invasive alternative method for detecting COVID-19 positive cases in a well-controlled cohort. ${ }^{28}$ Regarding the feasibility of the saliva self-sampling and testing, based on our periodic reports on the number of tests performed available on the university website ${ }^{31}$ and the high sensitivity and specificity of saliva testing, ${ }^{27}$ there is strong evidence that valid samples and reliable testing results will be obtained given that saliva massive testing among students and staff members in the researched context has been in place since the academic year 2020-2021. In other words, the practices have been an indispensable part of the institutional effort to manage the viral transmissions. Through these exercises, we have ensured that a cohort of informed participants, testing infrastructures and adequate supporting staff are present and ready to engage in this prospective longitudinal study. This is what distinguishes the research from other studies aimed to examine COVID-19 prevalence, incidence and immune response, which are usually conducted by convenience sampling or over a short period of time. Nevertheless, from a statistical point of view, roughly one-thirds of the staff members would be required to enable reliable generalisations for the ULiège's staff population. Despite certain logistic arrangements to ease the participation, it is believed that reaching this cohort might entail more challenges due to the complicated work-life circumstances. Therefore, it is probable that strategies encouraging staff's participation will be modified during the course of the study when we are more informed about hindrances encountered.

The prospective design of SARSSURV allows to derive reliable estimates of the prevalence, the incidence of COVID-19 in both symptomatic and asymptomatic participants, characterising the immune response while accounting for vaccine and/or previous infection a in higher education population. While using of serological self-test provides information about past COVID-19 infection, saliva self-test allows the detection of current 
infections. Combined, respectively, with a previous selfreported COVID-19 infection and with the collection of current symptoms, various COVID-19 profiles of interest can be derived. In addition, the longitudinal study design enables to derive these results at different stages of the disease as well as seroreversion and seroconversion after vaccination. On this basis, our knowledge about COVID-19 infection and seroprevalence among different participants' profiles, particularly among symptomatic/ asymptomatic and vaccinated/non-vaccinated participants, will be substantially enriched. Moreover, informed decisions as to COVID-19 measures and resuming of activities are further strengthened.

Regarding COVID-19 vaccination hesitancies, both university populations, namely students and staff members, are interviewed at different timepoints in order to account for the various information and attitudinal changes related to the evolution of the sanitary and vaccine situation in Belgium. Just like anamnestic and clinical questionnaire, vaccine hesitancy questionnaires are placed on an online platform to be completed by the participants, hence, minimising social desirability bias.

SARSSURV study has certain limitations. The inclusion of participants may be biased by their engagement against COVID-19, which may result in low secondary infection as participants could take more precaution against COVID-19 transmission. Although instructions were provided to the participants using detailed written procedure and available videos, the use of self-tests may lead to inconsistent results. To address this issue, effort will be invested to make results available as soon as possible so that new samplings will be collected if necessary.

Because of the longitudinal design of the study, and despite a recall system in place, the presence of intermittent missing data and drop-out are unavoidable given the workload of the staff members and the intermediate holidays and breaks observed in a higher education context. This might have a consequence on the plan to periodically monitoring of immune responses of vaccinated and/or infected participants and to ensure equal time intervals of symptom, saliva and blood sample collection for all participants. Given the prospective nature of the study, it is acknowledged that this is an inherent limitation that we should tackle so that timing should not be a confounding factor. For example, we explicitly request the participants to inform the research team if they have the intention not to continue their participation during the holidays. In so doing, we could be more proactively in the data collection process and improve our upcoming new recruitment plan.

Even if it is still unclear whether transmission of SARSCoV-2 is impacted by climatic effects, ${ }^{32}{ }^{33}$ as the present study is planned at least until June 2022, seasonal effect can be observed in the level of detectable infections. Because of the voluntary nature of participation, it is likely that not all strata are equally represented. However, this can be accounted for by different weighting procedures. For example, weights are assigned to the samples so that they mimic the ULiège population in terms of the age, gender and status (staff and students) by comparing the frequencies obtained from the sample and those from 2020.

As a conclusion, SARSSURV is a unique opportunity to obtain reliable epidemiological data related to the spread of SARS-CoV-2 (and its variants) in a university cohort and to better understand immunity against COVID-19 infection in a young population composed of student participants, as well as in adult staff members. In addition, the evolution in COVID-19 vaccination hesitancies may help to mobilise ULiège members as citizen health partners to develop skills and take informed decisions about COVID-19 vaccine. In reaching these endpoints, the protocol presented and the results to be obtained would be valuable to advance current understanding of the COVID-19 pandemic dynamics, research in the field of epidemiology and enable more effective public health interventions and protection.

\section{Author affiliations}

${ }^{1}$ Biostatistics Unit - Public Health Department, Liège University, Liège, Belgium ${ }^{2}$ Human Genetics Department, Centre hospitalier universitaire de Liège, Liège, Belgium

${ }^{3}$ COVID-19 Platform of University of Liège, Liège University, Liège, Belgium ${ }^{4}$ Infectious Diseases Department, Centre hospitalier universitaire de Liège, Liège, Belgium

${ }^{5}$ Department of Pathology, FARAH, Liège University, Liège, Belgium

${ }^{6}$ Laboratory of Cellular and Molecular Immunology, GIGA Institute, Liège University, Liège, Belgium

${ }^{7}$ Récolte et Analyse de Données et d'Information d'Utilité Stratégique (RADIUS), Liège University, Liège, Belgium

${ }^{8}$ Public Health Department, Liège University, Liège, Belgium

${ }^{9}$ Fundamental and Applied Research for Animal and Health (FARAH) Center, Liège University, Liège, Belgium

${ }^{10}$ Laboratory of Immunology-Vaccinology, FARAH, Liège University, Liège, Belgium

Acknowledgements We would like to thank all the students, staff members and laboratory staff of the University of Liège who dedicated their time to participate in this longitudinal study.

Contributors FB and LG are the principal investigators of the study. A-FD, MG and AND designed and drafted the study protocol. NG, MP, EH, A-FD and MG detail the field work and with $D D, V B, M D, A T, L F, C S$ and $L G$ conceptualized the laboratory work. EH is responsible for the conception of all IT-related study components, and together with NG, FM coordinate all the conception, design and operation of all ITrelated study components. NG, MP, FM and MD are in charge of data management and acquisition. All authors have read, substantively revised the study protocol and approved the submitted version of the manuscript. LG and FB are joint last authors.

Funding The authors have not declared a specific grant for this research from any funding agency in the public, commercial or not-for-profit sectors.

Competing interests The two last authors are the inventors of the device used in the saliva collection kit. This device was patented (EP20186086.3) and produced by Diagenode (Seraing, Belgium) under a commercial agreement with the University of Liège.

Patient and public involvement Patients and/or the public were not involved in the design, or conduct, or reporting, or dissemination plans of this research.

Patient consent for publication Not applicable.

Provenance and peer review Not commissioned; externally peer reviewed.

Open access This is an open access article distributed in accordance with the Creative Commons Attribution Non Commercial (CC BY-NC 4.0) license, which permits others to distribute, remix, adapt, build upon this work non-commercially, and license their derivative works on different terms, provided the original work is properly cited, appropriate credit is given, any changes made indicated, and the use is non-commercial. See: http://creativecommons.org/licenses/by-nc/4.0/. 


\section{ORCID iDs}

Anne-Françoise Donneau http://orcid.org/0000-0002-5385-6289

Anh Nguyet Diep http://orcid.org/0000-0001-5134-3220

\section{REFERENCES}

1 Sohrabi C, Alsafi Z, O'Neill N, et al. World Health Organization declares global emergency: a review of the 2019 novel coronavirus (COVID-19). Int J Surg 2020;76:71-6.

2 Johns Hopkins University. COVID-19 dashboard by the center for system science and engineering (CSSE) at Johns Hopkins University (JHU). In Johns Hopkins Coronavirus Resource Center [Online], 2020. Available: https://coronavirus.jhu.edu/map.html [Accessed 6 Jul 2021].

3 Federal Public Service for Health, Food Chain Safety and Environment. 6 new cases of Covid-19 by the end of the spring holidays [Online]. Available: http://info-coronavirus.be [Accessed 11 Jun 2021].

4 Statistica. Coronavirus (COVID-19) deaths worldwide per one million population as of July 6, 2021, by country, 2021. Available: https:// www.statista.com/statistics/1104709/coronavirus-deaths-worldwideper-million-inhabitants [Accessed 6 Jul 2021].

5 Saegerman C, Bianchini J, Renault V, et al. First expert elicitation of knowledge on drivers of emergence of the COVID-19 in pets. Transbound Emerg Dis 2021;68:626-36.

$6 \mathrm{Bi} \mathrm{Q}, \mathrm{Wu}$ Y, Mei S, et al. Epidemiology and transmission of COVID-19 in 391 cases and 1286 of their close contacts in Shenzhen, China: a retrospective cohort study. Lancet Infect Dis 2020;20:911-9.

7 Burke RM, Midgley CM, Dratch A, et al. Active monitoring of persons exposed to patients with confirmed COVID-19 - United States, January-February 2020. MMWR Morb Mortal Wkly Rep 2020;69:245-6.

8 Weeden K, Cornwell B. The small-world network of college classes: implications for epidemic spread on a university campus. Sociol Sci 2020;7:222-41.

9 European Centre for Disease Prevention and Control. COVID-19 testing strategies and objectives, 2021. Available: https://www.ecdc. europa.eu/en/publications-data/covid-19-testing-strategies-andobjectives [Accessed 6 Jul 2021].

10 Rothe C, Schunk M, Sothmann P, et al. Transmission of 2019-nCoV infection from an asymptomatic contact in Germany. N Engl J Med 2020;382:970-1.

11 Randad PR, Pisanic N, Kruczynski K. COVID-19 serology at population scale: SARS-CoV-2-specific antibody responses in saliva. J Clin Microbiol 2021;59:e02204-20.

12 Stadlbauer D, Tan J, Jiang K, et al. Repeated cross-sectional sero-monitoring of SARS-CoV-2 in New York City. Nature 2021;590:146-50.

13 Tan SH, Allicock O, Armstrong-Hough M, et al. Saliva as a goldstandard sample for SARS-CoV-2 detection. Lancet Respir Med 2021;9:562-4.

14 Kapoor P, Chowdhry A, Kharbanda OP, et al. Exploring salivary diagnostics in COVID-19: a scoping review and research suggestions. BDJ Open 2021;7:8.

15 Butler-Laporte G, Lawandi A, Schiller I, et al. Comparison of saliva and nasopharyngeal swab nucleic acid amplification testing for detection of SARS-CoV-2: a systematic review and meta-analysis. JAMA Intern Med 2021;181:353-8.

16 Wyllie AL, Fournier J, Casanovas-Massana A, et al. Saliva or nasopharyngeal swab specimens for detection of SARS-CoV-2. N Engl J Med 2020;383:1283-6.

17 University of Liege. In Infos coronavirus, 2021. Available: https:// www.coronavirus.uliege.be/cms/c 13565085/en/study-of-anti-sarscov-2-covid-19-seroprevalence-at-the-university-of-liege-sarssurv [Accessed 6 Jul 2021].

18 Larson HJ, Jarrett C, Schulz WS, et al. Measuring vaccine hesitancy: the development of a survey tool. Vaccine 2015;33:4165-75.

19 Betsch C, Schmid P, Heinemeier D, et al. Beyond confidence: development of a measure assessing the $5 \mathrm{C}$ psychological antecedents of vaccination. PLoS One 2018;13:e0208601.

20 Goldstein S, MacDonald NE, Guirguis S, et al. Health communication and vaccine hesitancy. Vaccine 2015;33:4212-4.

21 University of Liege. In test de screening COVID-19, 2021. Available: https://test-covid.uliege.be/testcovid/index_xt.do [Accessed 6 Jul 2021].

22 University of Liege. In how do I get my self-collection kit? 2021. Available: https://www.coronavirus.uliege.be/cms/c_12288701/en/ how-do-i-get-my-self-collection-kit [Accessed 6 Jul 2021].

23 Hacisuleyman E, Hale C, Saito Y, et al. Vaccine breakthrough infections with SARS-CoV-2 variants. $N$ Engl J Med 2021;384:2212-8.

24 Diep AN, Gilbert A, Saegerman C, et al. Development and validation of a predictive model to determine the level of care in patients confirmed with COVID-19. Infect Dis 2021:53:590-9.

25 Docherty AB, Harrison EM, Green CA, et al. Features of 20133 UK patients in hospital with COVID-19 using the ISARIC WHO Clinical Characterisation Protocol: prospective observational cohort study. BMJ 2020;369:m1985.

26 Saegerman C, Gilbert A, Donneau A-F, et al. Clinical decision support tool for diagnosis of COVID-19 in hospitals. PLoS One 2021;16:e0247773.

27 Tonen-Wolyec S, Dupont R, Batina-Agasa S, et al. Capillary wholeblood lgG-IgM COVID-19 self-test as a serological screening tool for SARS-CoV-2 infection adapted to the general public. PLoS One 2020;15:e0240779.

28 Ministère des Solidarités et de santé. In Tests COVID-19, guider votre choix parmi les 155 tests COVID-19 du marché [Online], 2020. Available: https://covid-19.sante.gouv.fr/tests [Accessed 20 Jun 2020].

29 Daniel WW, ed. Biostatistics: a foundation for analysis in the health sciences. 7th ed. New York: John Wiley \& Sons, 1999.

30 von Elm E, Altman DG, Egger M, et al. The strengthening the reporting of observational studies in epidemiology (STROBE) statement: guidelines for reporting observational studies. PLoS Med 2007; 4:e296.

31 University of Liege. In Dasbhoard results, 2021. Available: https:// www.coronavirus.uliege.be/cms/c_14106329/fr/coronavirusresultats-du-tableau-de-bord [Accessed 16 Nov 2021].

32 Engelbrecht FA, Scholes RJ. Test for Covid-19 seasonality and the risk of second waves. One Health 2021;12:100202.

33 Merow C, Urban MC. Seasonality and uncertainty in global COVID-19 growth rates. Proc Natl Acad Sci U S A 2020;117:27456-64. 